\title{
Organizational reconciliation and its implications for organizational decision support systems: a semiotic approach
}

\author{
Jonathan Liebenau ${ }^{\mathrm{a}}$, G. Harindranath ${ }^{\mathrm{b}, *}$ \\ ${ }^{a}$ Department of Information Systems, London School of Economics, University of London, Houghton Street, London WC2A $2 A E$, UK \\ ${ }^{\mathrm{b}}$ School of Management, Royal Holloway, University of London, Egham, Surrey TW20 OEX, UK
}

Accepted 1 October 2001

\begin{abstract}
This paper describes the key problems with the core concepts behind ODSS, and provides a critique from a theoretical perspective that draws on organizational theory and semiotics. We argue that any distinction between organizational decision support systems (ODSS) and other related systems such as DSS or group decision support systems (GDSS) should be based on the difference between the characteristics of groups and organizations. Our approach uses characteristics of communication within organizations to model information in order to support organizational level decisions. We illustrate the usefulness of this approach by examining a decision-making problem in the context of a British hospital. (C) 2002 Elsevier Science B.V. All rights reserved.
\end{abstract}

Keywords: ODSS; DSS; Organizational theory; Semiotics

\section{Introduction}

Organizational decision support systems (ODSS) are a class of decision support systems that promise to provide support at a higher organizational level for businesses than preceding forms of decision support. The existing literature provides many different descriptions of ODSS and its functionality. This leads to considerable confusion as to what is necessary for a system to be called an ODSS. This confusion has inhibited the development of ODSS both conceptually and in terms of implementation. We begin by considering the existing conceptual base upon which ODSS

\footnotetext{
${ }^{*}$ Corresponding author.

E-mail addresses: J.L.Liebenau@1se.ac.uk (J. Liebenau), g.harindranath@rhul.ac.uk (G. Harindranath).
}

is built and propose a means of distinguishing organizational decisions from executive and group decisions. Several different conceptions of ODSS have been provided in the literature but all of them are essentially modifications of group decision support systems (GDSS). In so far as there is a distinction, it should be based on the difference between the characteristics of groups and organizations. People within groups share norms and typically work toward consensus building through decision making, while the key characteristic of heterogenous organizations is that they require assistance to reconcile divergent perceptions as their first step toward decision making.

The purpose of the conceptualization is to provide the means to build upon GDSS functionality in a way that takes organizational features seriously. This may come in the form of better interfaces or different 
functionality, but in essence, it is the emphasis on the organizational features of hierarchy and heterogeny that will make ODSS advantageous. Our semioticsbased approach will focus on these characteristics and provide a means of using the analysis of organizational communications to model information to support organizational decision-making. The next section presents various definitions of ODSS. This is followed by a consideration of the key differences between groups and organizations. Next, the semiotic approach we take will be explained in more detail. The paper ends with a hospital-based example that shows how the semiotic approach can be beneficially used to identify and refine organizational level problems that are suitable for ODSS support.

George [7] summarized the conceptualization and development of ODSS and emphasized the way the organizational perspective was first introduced into the domain of decision support. The opportunities afforded by data communication and the renewed emphasis on cheaper business computing created the means to integrate decision support to include first small groups of decision makers (as in GDSS) and, later, many different groups (as in ODSS).

In its earliest conceptualization, the primary emphasis of ODSS was upon the communication and coordination function $[3,8]$. The subsequent history of decision support system developments in the 1980s rests on the difference between technology- and business-driven approaches. DSS approaches were seduced by technology-driven opportunities afforded by personal computers, network computing, and related software developments. Because technology-driven approaches became the mainstream, all efforts were directed to exploiting these new functionalities at the expense of a focus on organizational problem solving.

Although GDSS and ODSS are types of multiparticipant [9] decision support systems, we agree with King and Star [14] who claim that we cannot simply scale up from DSS to GDSS or from GDSS to ODSS. They imply that there are more than contextual differences between these different kinds of systems because group representatives tend to operate in an organizationally sub-optimal way as their first loyalty is to their own groups rather than to the organization.

However, Miller and Nilakanta [19] do regard DSS and ODSS to be closely related: "Note that the difference between DSS and ODSS is in the context of the decision and it is believed that much of the technical design aspects of ODSS (e.g., data and model management capabilities and the interfaces among the subsystems) will not be very different." Aggarwal and Mirani [1], however, propose a broader definition of an ODSS which includes all systems that provide "borderless" and "seamless" decision-making support across functional, divisional, and national boundaries.

The technological features of these different types of decision support systems are exemplified by the difference between single machine operations, which is the minimal necessary unit for DSS, a decision room which is typical for GDSS, and the possibilities of dispersed computers, perhaps even spread among different organizations, which can be said to characterize ODSS. The application of these concepts to the architectures of multiparticipant decision support systems, in general, can be seen in the work of Holsapple and Whinston [10]. Similarly, Jacob and Pirkul [11] address the organizational perspective from the point of view of multiparticipant decision making which can be structured around nodes within networks of knowledge-based systems. Each of these relies upon different technical structures, but all are implicitly intended to serve the business functions. What are the real differences beyond this manifestation? To make the various forms of decision support truly distinct, we should look beyond the technical features of these operating architectures.

If we see the original purpose of ODSS as emphasizing communication and coordination, we should regard this as addressing the problem of reconciliation. Later work by Weisband and Galegher [24] emphasized the need to address organizational diversity and flexibility, which we can regard as addressing the problem of organizational heterogeny. We propose that organizational communication and its attendant problems of reconciliation, along with heterogeny, constitute the two domains which ODSS should address. We will now examine the significance of these two organizational features vis-a-vis the characteristics that are typical of groups in relation to organizations.

\section{Groups and organizations}

Groups meet with the belief that they have sufficient understanding of the goals and assumptions of 
their distinct problem-solving environment. We can refer to these assumptions and understandings as 'norms' and this allows us to apply norm-based analyses upon group decision-making processes. The most important function of a GDSS would be to build consensus so that the shared norms can effectively be used in the decision-making. The architecture and software capabilities of GDSS emphasize this importance. Much effort is given to allow for voting-type behavior and other group consensus techniques. Similarly, the software supports the work of a group moving through the draft stages of a text. Some GDSS are especially good at providing techniques, usually through well-designed interfaces and presentational graphics, to promote focused discussion.

Groups usually agree that key organizational issues such as strategy, structure, and boundaries are given. They are usually concerned with operational level decisions such as procedures, production techniques, and business efficiency. Pinsonneault and Kraemer [21] report on a number of experimental and other empirical works which show how "GDSS focus the attention and efforts of group members on task-related activities", and refer to findings which show increased depth of analysis, task-oriented communication, and clarification efforts. They conclude that these results demonstrate the efficacy of GDSS in increasing consensus in groups.

Organizations, in contrast to groups, are usually heterogenous. So the most important function, which any computer system that supports decision-making can do, is to recognize the common requirements of different groups, and to reconcile different perceptions.

Organizations generally behave in ways different from groups. We can take the example of the Canadian telecommunications company described by Marche $[17,18]$, which, in trying to determine what a building is, illustrates the differences which arise among groups when an organization tries to reconcile norms. In this company, a seemingly straightforward effort to model data about corporate real estate holdings revealed extraordinary difficulties when it was clear that each group within the organization needed to conform to conflicting ontologies. For the accountants, a building could be defined as something that was depreciating. For the planners responsible for assigning space to people, a distinct building was something that could be conceived as a contiguous space. The architects saw the building in planning terms. Others were concerned with problems such as the definition of temporary or mobile structures. So many cases arose which yielded entirely different solutions, and Marche gives the example of a building which, having been originally erected in 1958, extended in 1969, and further built upon in 1985, was seen by different groups to be one, two, or even three buildings.

As we can see in the example above, organizations are required to integrate differing functions, usually within hierarchies, for common goals. Inherently, organizations transcend groups. When organizations make decisions, they must take into account that different perceptions are brought in by different interested persons. Each organizational component brings with it a different set of norms. This heterogeny is the result of a variety of functionalities for which organizations take responsibility. For instance, they need to accommodate widely divergent functions such as the production, distribution and sale of goods, the maintenance of solid legal footing, and the requirements of accounting activities. Without this heterogeny, this necessary variety cannot function effectively.

Many of the differing perceptions brought in by heterogeny will conflict with one another, despite an overall shared organizational goal (i.e., the purpose of the business). What is required for effective ODSS will then be a means to analyze these differing perceptions and to provide the tools to allow participants to recognize mutually acceptable approaches to reach common goals. The semiotic approach to information can provide one such tool.

\section{A semiotic approach to organizations}

A semiotic approach to information systems provides a tool to represent organizational knowledge and activity. The theory of signs originates in the work of Peirce [20] who shows that a sign must be capable of evoking responses from the interpreter or a person. Semiotics makes us recognize the importance of an agent as being responsible for the existence of a sign and its meaning. Organizations can be seen as complexes of signs communicated among people who, acting as semiotic agents, are responsible for assigning meaning $[2,6,12,15,17,18,23]$. 
This approach provides a conceptual breakdown of information systems into four levels. This helps us to identify the range of issues from the cultural environment and problems of meaning to formal structures and the physical characteristics of codes and signals [16]. Level one, called 'pragmatics', is concerned with the actual context of activity and those characteristics of people, organizations, and acts of communication which affect information. Shared assumptions among people, and problems arising from ambiguities in communications, and the informal nature of most organizational interactions form the concern of pragmatics. 'Semantics' and tools of semantic analysis, which form level two of the semiotic framework, deal with problems of meaning. They also help us create formalizable models of networks of meaning in organizations. Level three, termed 'syntactics', deals with the use of formalisms, and level four, 'empirics', is concerned with the physical characteristics of codes and signals.

There are other structured and semi-structured techniques, which also provide analysts with tools to interpret the uses of information within organizations. However, approaches such as Peter Checkland's [5] "soft systems methodology" are intended as means to facilitate systems design by eliciting user requirements. Other more formal approaches seek to associate efficient data flow structures with organizational structures. Though they may be useful for systems design, they are unable to provide a representation of organizational activity.

Cecez-Kecmanovic [4], in proposing a conceptualization of what she calls organizational activity support systems, uses a semiotic approach to the problem. According to her, a semiotic approach "recognizes organizational entities and their respective roles, persons as role holders, patterns of actions and types of activities as well as documents and information, and their flows within an activity and between activities" [4]. Further, the approach is based on the principle that different kinds of knowledge are assumed in the performance of activities in an organizational context. Cecez-Kecmanovic demonstrates a technique of knowledge representation, which captures these dimensions and show their interrelationships. She goes one step further by creating a formal model of organizational activity (i.e., she creates schemas/models of organizational entities, roles, personnel and their activities, documents and data; these models can then be used to simulate solutions to organizational decisionmaking problems). Such a model of organizational activity is fundamentally based on the semiotics of organizational communications and upon speech act analysis. Her formalization convincingly demonstrates that semiotic techniques can provide one pillar of support for ODSS design.

Now let us consider how we can bring together our understanding of the organizational problems that ODSS can ameliorate with the semiotic technique. We do this by separating our approach into the distinct concepts which we call 'prioritization', 'contextualization', 'formalization', and 'building in functionality'. These are drawn from the semiotic levels described above, 'pragmatics', 'semantics', 'syntactics', and 'empirics'. First, we will have to clearly specify the tangible problems that people face at the organizational level.

\subsection{Prioritization: identifying 'organizational level' problems}

Keen [13] identifies a number of needs for DSS research and lists first the question, "What decisions really do matter in an organization and how should we build better environments to help decision makers handle them?" For the purposes of this argument, let us identify three typical decision problems that must be addressed at the organizational level: boundaries, structure, and strategy.

What constitutes the organization and its boundaries is increasingly problematic, because of the extensive interlinkages among organizations and the rapidly growing opportunities which businesses have to outsource common functions. Most large organizations recognize the need to decide on which functions should be internalized and which ones outsourced. Similarly, the boundaries between linked organizations need to be continuously negotiated.

Another typical problem that must be solved at the organizational level concerns the internal structure that the organization should have. Companies regularly reassess the shape of their hierarchies and attempt to maximize the opportunities offered by new organizational forms such as networked or distributed structures.

A third set of organizational concerns comes under the category of business strategy. This involves the 
decision upon how resources are allocated to advantageously position the firm for future opportunities. True strategic decisions implicate the whole organization and not only subsets thereof (although such decisions may be made by the top management). Decisions of these kinds are especially appropriate for ODSS. These are the kind of problems that ODSS should be focusing on, precisely because they are organizational issues as distinct from the kind of problems which groups within organizations typically have to encounter.

\subsection{Contextualization: understanding why percep- tions of organizational level problems differ}

Having examined some of the most important organizational level problems, we need to understand why different groups have conflicting perceptions of these concerns. We can do this by making the 'organizational' characteristics of these problems explicit through the use of organizational theory and semiotics.

There are some recent efforts to incorporate elements of this approach into ODSS, although they need to be more explicitly grounded in organizational theory. One good case is presented by Miller and Nilakanta [19] in their example of the Boone Cannery. As the cannery extended its business and added several trading partners, EDI made it possible for the firm to extend its information handling tasks. However, the key decisions that had to be taken affected a number of people at various functional and hierarchical levels. In addition to procedural matters, "organizational norms and policies need to be enforced or changed. At the departmental or functional unit level, inter-unit communication becomes a necessity. Communication infrastructures and protocols must be established so that conflicts are reduced. Because of self preservation tendencies, organizational units may suboptimize or act in discordance with the overall objective of the firm [19]." Different groups may have conflicting perceptions of the abovementioned problems because their interests vary. They may also be characterized by varying levels of access to information, and differences in their ability to understand issues that span the entire organization. Semiotics and, in particular, the notions of pragmatics and semantics within it, provide a means to examine these issues. Pragmatics identifies different kinds of organizational norms. Semantics provides a preliminary step to the presentation of semantic schema which are based on norm analytic structures. Both norm analysis and schema design are well-recognized techniques of the semiotic approach that can be used to analyze and model divergent organizational views $[2,16]$.

The purpose of drawing schema is to capture the relationships between those elements that make up the entirety of the system being examined. Also, by drawing the schema, the analyst is forced to assign unique relationships among elements. This discipline assists in revealing ambiguities and reconciling conflicts. To do this, we must at least capture the basic

\section{ontological dependency line hash for individuation period for part/whole \\ joint affordance or relationship role in relationship ()}

sign $\left[{ }^{*}\right]$ for realisation dotted line [--] for referent

generic/specific box
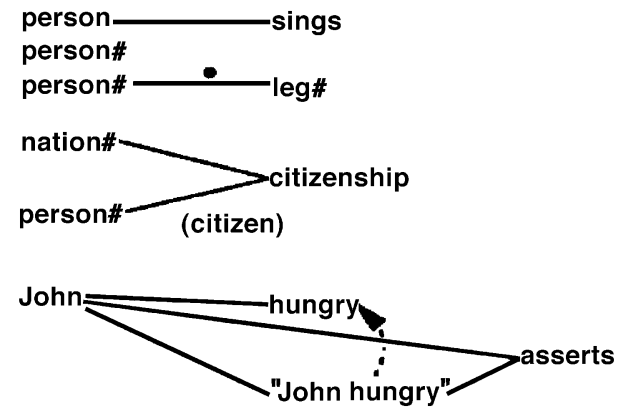

fruit

apple

etc.

Fig. 1. Graphical notation symbols for semiotic shemata, taken from [Ref. [2], p.84]. 
characteristics of relationships. These are: the relationship which shows how one element is dependent on another to exist, called "ontological dependency"; the specification of an individual; the relationship between parts and wholes; the roles in relationships; and the relationship between specific and generic characteristics. Fig. 1 describes one notational system for drawing semantic schema and we shall use this in an example in the next section.

\subsection{Formalization: modeling of divergent organiza- tional perceptions}

The next step is to be able to analyze and model divergent organizational perceptions. This can be accomplished through the approach used by CecezKecmanovic [4] to model organizational communications. Once these divergent perceptions are formalized, they can then be manipulated. This is an essential step towards building in functionality.

\subsection{Building in functionality}

Once the various organizational perceptions have been identified and modeled, we need to be able to build in the necessary functionality as Miller and Nilakanta [19] do in their designing of a data extraction scheme for ODSS.

While the work of Cecez-Kecmanovic [4] and that of Miller and Nilakanta [19] are important and useful in themselves, their scope is limited. The scope of Miller and Nilakanta's work is limited because they are primarily interested in data-handling issues. The key issues that emerge from their work are at the level of norms. Cecez-Kecmanovic's work takes this approach further towards a formalizable model and shows how it can be used in the designing of ODSS. Her work takes a semiotic approach to the problem of creating formal models of communications based on the norm structures of organizations. Thus, we could say that Cecez-Kecmanovic's work has formalization without functionality, whereas Miller and Nilakanta provide us with this functionality without explicitly relating it to organizational theory. Without clear links to organizational theory, their work does not sufficiently differentiate organizational level and group level issues. We therefore need to place the issue of ODSS in perspective and locate it within organiza- tional theory using semiotics and, in particular, on distinguishing between organizational and group level problems.

Now the challenge is to use the semiotic technique in organizational analysis to satisfy the requirements of prioritization (of organizational level decision problems), contextualization, formalization, and functionality. This could go some way towards providing a clearer conceptualization of organizational decision problems and, therefore, what an ODSS should actually be. In the following section, we demonstrate its utility through an example.

\section{Organizational decision making in a British hospital: staff scheduling for ambulatory care}

Let us take the case of decision-making in a British hospital. This hospital has embarked on building an ambulatory care and diagnostic center and the staff scheduling decisions there lend themselves to consideration as an organizational decision-making model. The purpose in using this example is to demonstrate the continuity of conceptual, as well as functional elements, through the whole of the analytical process. It must hold its integrity through the processes of determining the priorities, interpreting the context, designing the system, and ensuring that it functions in a way which satisfies all of the interested parties.

Ambulatory care is distinct from emergency and long-term in-patient care. The hospital's expectation is to increase the number of patients and improve the quality and access to service. The intention is also to make considerable financial savings over existing hospital practices. One of the key features of this distinction is that staff members are allocated differently from previously, where a third work schedule is required, in addition to that for emergency coverage and normal ward duties. A number of departments will move to the new center and this will involve substantial differences in working practices for medical staff, and different access to treatment and diagnosis for patients. The center will depend entirely on effective scheduling services. This dependence can be exemplified by the substitution of normal reception services with highly skilled people that are able to assess individual treatment needs and their staffing requirements [22]. 
Here, we take the requirements of the staff scheduling problem and apply our approach to ODSS using organizational theory.

\subsection{Prioritization}

The new center needs to have systems that can cope with referrals from, and other relationships with, the existing health care institutions. This will include other hospitals, general practitioners, clinics, private health care providers, and other elements of the health system. This not only defines the center's boundary in relation to the main hospital, it also establishes its procedures in relation to other bodies which may wish to use or be used by the center. One of the key features of ambulatory care is that the entire procedure, which is to be applied towards patient care, should be reduced from typically 4-10 days to 1 day. All other forms of care must be referred elsewhere. These features of boundaries must be designed into the operating systems in use elsewhere, such that scheduling and staffing arrangements can be seamlessly integrated into the main hospital systems. A clear definition of the boundary expresses not only the distinctiveness of the center; it also accommodates its interrelationships. All features of the information system are affected at the outset by these decisions about where the boundaries are.

In this example, we will consider one organizational level decision problem, the process of scheduling treatment. This example will illuminate a number of features that can be regarded as touching upon the boundary of the organization, its structure, and to some extent, its strategy. Boundary issues arise with respect to the relationship between the patient and the general practitioner in so far as the patient has links to both the general practitioner and the center. Boundary issues also arise with respect to the relationship between the patient and the medical staff who may work for the center as well as other clinics. Structural issues arise in the relationships among key members of the staff, and, in particular, are idiosyncratic in the manner in which schedulers control the working time of doctors. As one of the key purposes of the center is to maximize patient throughput, this all-encompassing concept of scheduling is vital for the center's strategy because it is this feature which distinguishes it from other forms of medical institutions. The architecture of the system must replicate the network of relations among local health providers and distinguish between in-patient, out-patient, and community care status.

In short, the purpose of the center is to reform the whole of the organization's position such that strategic decisions are built into its operation. These would include specialization decisions about general versus special hospital services. It must also establish the clear division of services, such that ambulatory versus emergency coverage is kept distinct. It is also exemplified in financial structures: managing private capital in relation to publicly supported running costs.

\subsection{Contextualization}

Once we have determined the organizational level problems, we need to contextualize them through an interpretation of the key norms of behavior which apply, as well as an analysis of the context as understood through the use of language and its semantic content. In the case of the center, there are the general medical norms associated with the Hippocratic ideals. There is also the overriding health service concern with affordable medicine, and the political commitment to equitability. Other salient norms include the practices of communication, which occur within medical hierarchies. These are enshrined in the manner in which orders are transmitted, reports are shared, and authority is exercised. Overall, the system needs to recognize the timeliness, urgency, and the quality that is required for dispensing medical care. When these norms are specified and realistically elaborated, they can be used as the basis of a semantic schema, which will then serve as a model of how a scheduling decision is made and may be partly incorporated into any automated system.

When we take a scheduling problem for staff allocation in the center, then we are concerned with how many patients can be seen in the clinic. The intention is to maximize the throughput of patients, and when each patient arrives, the scheduling procedure begins with an assessment of how long it will take to diagnose and then to treat the malady. This involves not only the assessment of the patient's apparent condition, it also needs to take the available medical staff into account. Each physician works to their own schedule, and that schedule must correspond to the available support staff and facilities. If, for example, a 


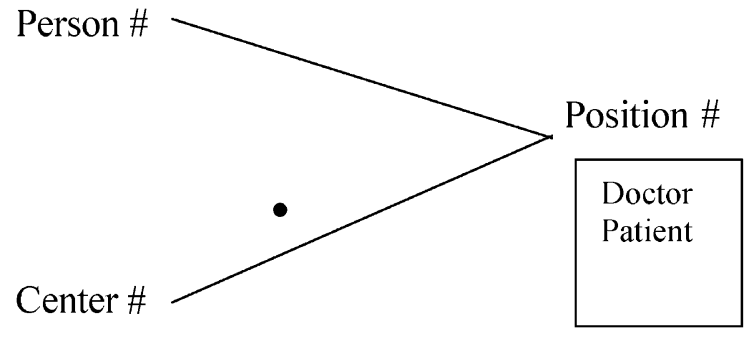

Fig. 2. Schema 1 -The relationship between the ambulatory care center and doctors and patients.

patient comes in needing an operation, the scheduler will have to assess the amount of time needed for a full diagnosis, the time and facilities needed for the preparation for surgery, and the full staff and facilities required for the surgery itself. The patient requires timely treatment, but the entire system requires accurate assessment so that each entire day of activity involving dozens of patients, many medical staff members, and a few scarce facilities are all taken into account together. Beyond the need for straightforward efficiency, the schedule will take the feelings and preferences of staff members into account, it might consider whether a doctor has had an especially heavy work load that day, etc.

In drawing the schema, the affordances of each of the following need to be considered: general practitioners who provide a preliminary diagnosis and a referral; schedulers who schedule diagnostic work and treatment and also inform patients and staff (they also schedule staff and facilities); doctors who diagnose and treat; nurses and ancillary medical staff who assist doctors and treat; and patients who receive treatment. We note that patients become patients from the time they are given a preliminary diagnosis by their GP. They become patients of the center from the time they are referred to the center. They remain as patients through the period of their treatment, after which they become "former patients". Facilities have temporal characteristics (e.g., their availability to patients and their doctors) and these need to be taken into account in the schema.

Each relationship should be described in a manner that allows us to understand the features that are required for the existence of the associated features. As we do this, we reveal ambiguities and reconcile contradictions. The schema should also adequately represent a number of other elements and activities that form part of the scheduling and treatment process. These would include the following: messages about the patient's referral; the final report (one form of which goes to the patient, another to his general practitioner); patient record (starts when the patient is referred and is added to with each action); notification of appointment; patient's reply to the notification (accepting or rejecting the schedule; or implicitly accepting); patient's arrival at the center and is later given notification (via a paging system); patient's arrival at the facility for treatment; treats; doctor's dictation notes for the report on treatment which is added to the patient record; and load (for doctors, other staff, and facilities).

For instance, Schema 1 in Fig. 2 shows the relationship between the ambulatory care center and doctors and patients. The individuation marks show that person, center, and position are particular occurrences that are interdependent. The link between position and the center is shown as a "part-whole" relationship because patients and doctors are associated also with other institutions.

Schema 2 in Fig. 3 extends this to show the status of a referral. Here, position and facility are shown with their necessary temporal characteristics.

\subsection{Formalization}

We can now model the relationships expressed in the schema in order to incorporate the normal fea-

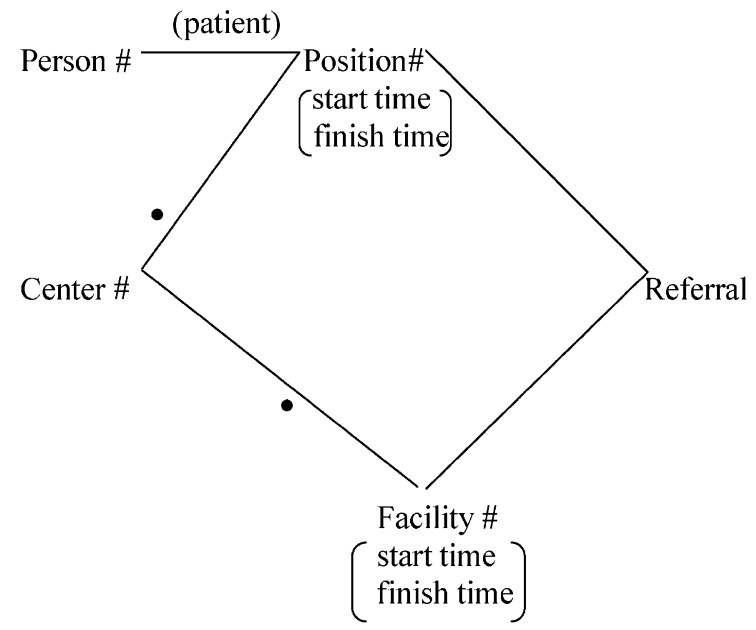

Fig. 3. Schema 2-A referral. 
tures of any data model. Extending Schema 2, we can formalize the referral system by establishing the significance of the links among persons and the organization. We then account for the temporal characteristics as well as the associations that prevail at the time when a referral occurs. Taking further the example of the British hospital, more extensive formalizations might include workload, periods of time, responsible agents, who schedules, what recourse to changes/objections, etc. Each of these elements needs to be schematically presented and then further formalized to create the necessary functionalities. Then, calculations associated with functions such as personnel allocation, load analysis, and scheduling can be performed.

\subsection{Building in functionality}

It is the above capacity to perform operations which provides us with the necessary functionality. As the formalization extends to include more and more relationships and more data handling operations, we can maintain, in an integrated manner, the entirety of the system. The functional design features of the new system need to accommodate institutional relationships. In the case of our example, these would include accommodating staff and patients from different sources; health care and hospital policies; institutional and professional norms; cost and financial features; acceptable communication mechanisms. These must be implementable and operable by knowledgeable managers.

The value of this, for the purposes of designing ODSS, is that we can distinguish between organizational and group level issues (prioritization) and work in an internally consistent manner from the structure of norms through semantic schema (contextualization) and to formalization.

\section{Conclusions}

In this paper, we have provided an approach that effectively differentiates organizational level decision problems from those at the group or individual level. We have also shown that the difference between ODSS and related systems such as DSS or GDSS, should be based on the difference between the characteristics of groups and organizations. The semiotic tool addresses the fundamental problem of reconciling differing perceptions within the organization to assist in overcoming the inherent problems of heterogeny. Previous works have either focused on providing functionality without explicit relation to organizational theory, or formalization without offering functionality. Our approach solves the problem of linking theoretical clarity with functional guidance.

The example of the ambulatory care center analysis shows how the concept of semiotics, applied to organizational theory, can provide the basis for identifying organizational level decision-making processes. By differentiating theoretically between group and organizational level problems, we can show situations where ODSS are useful. We can thus identify the distinctions between where DSS or GDSS function versus what we can expect ODSS to do. This approach can be used for identifying a class of problems that are located at the organizational level. It can also be helpful in demonstrating where more straightforward, DSS-based approaches might be sufficient, thereby avoiding an unnecessary diversion into organizational level decision making processes. By addressing organizational level problems as if they were at the level of the group, decision support systems are bound to be misapplied.

\section{References}

[1] A. Aggarwal, R. Mirani, Macro issues in the development of organizational decision support systems, Proceedings of the Twenty-eighth Annual Hawaii International Conference on System Sciences, IEEE Computer Society Press, Los Alamitos, CA, 1995.

[2] J. Backhouse, The use of semantic analysis in the development of information systems, PhD Thesis, London School of Economics and Political Science (1992).

[3] R.H. Bonczek, C.W. Holsapple, A.B. Whinston, Computerbased support of organizational decision making, Decision Sciences 10 (1979) 268-291.

[4] D. Cecez-Kecmanovic, Organizational activity support systems, Decision Support Systems 12 (1994) 365-379.

[5] P. Checkland, Systems Thinking, Systems Practice, Wiley, Chichester, England, 1981.

[6] G. Dhillon, Interpreting the management of information systems security, PhD Thesis, London School of Economics and Political Science (1996).

[7] J.F. George, The conceptualization and development of organizational decision support systems, Journal of Management Information Systems 8 (3) (1992) 109-125. 
[8] R.D. Hackathorn, P.G.W. Keen, Organizational strategies for personal computing in decision support systems, MIS Quarterly 5 (3) (1981) $21-27$.

[9] C.W. Holsapple, Decision support in multiparticipant decision makers, Journal of Computer Information Systems (Summer, 1991) 37-45.

[10] C.W. Holsapple, A.B. Whinston, Decision Support System: A Knowledge-based Approach, West Publishing, Minneapolis, 1996, pp. $599-651$.

[11] V.S. Jacob, H. Pirkul, Organizational decision support systems, International Journal of Man-Machine Studies 36 (1992) 817-832.

[12] M. Karamüftüoğlu, Knowledge Based Information Retrieval: A Semiotic Approach, PhD Thesis, City University, London (1998).

[13] P.G.W. Keen, Decision support systems: the next decade, Decision Support Systems 3 (1987) 253-265.

[14] J.L. King, S.L. Star, Conceptual foundations for the development of organizational decision support systems, in: Proceedings of the Twenty-third Annual Hawaii International Conference on Systems Sciences, vol. 3, IEEE Computer Society Press, Los Alamitos, CA, 1990, pp. 143-151.

[15] K. Kitiyadisai, Concepts of Relevance in a Semiotic Framework Applied to Information Systems Analysis and Design, $\mathrm{PhD}$ Thesis, London School of Economics and Political Science (1990).

[16] J. Liebenau, J. Backhouse, Understanding Information: An Introduction, Macmillan, Hampshire, 1990.

[17] S. Marche, On what a building might not be - a case study, International Journal of Information Management 11 (1991) $55-66$.

[18] M.M. Marche, Models of Information: The feasibility of measuring the stability of data models, $\mathrm{PhD}$ Thesis, London School of Economics and Political Science (1991).

[19] L.L. Miller, S. Nilakanta, Organizational decision support systems: the design and implementation of a data extraction scheme to facilitate model-database communication, Decision Support Systems 9 (1993) 201-215.

[20] C.S. Pierce, in: C. Hartehorne, P. Weiss (Eds.), Collected Papers, vols. 1-8, Harvard University Press, Cambridge, $1931-$ 1958 .
[21] A. Pinsonneault, K.L. Kraemer, The impact of technological support on groups: an assessment of the empirical research, Decision Support Systems 5 (1989) 197-216.

[22] J.A.A. Sillince, G. Harindranath, Integration of requirements determination and business process re-engineering: a case study of an ambulatory care and diagnostic (ACAD) centre, European Journal of Information Systems 7 (1998) $115-$ 122.

[23] R. Stamper, Research issues in information systems: semantics, in: R.J. Boland, R.A. Hirschheim (Eds.), Critical Issues in Information Systems Research, Wiley, Chichester, 1987.

[24] S.P. Weisband, J. Galegher, Four goals for the design of organizational information support systems, Proceedings of the Twenty-third Annual Hawaii International Conference on Systems Sciences, vol. 3, IEEE Computer Society Press, Los Alamitos, CA, 1990, pp. 137-142.

Jonathan Liebenau is Senior Lecturer in Information Systems at the London School of Economics and concentrates on two areas of research and teaching: fundamental concepts of information and the social, economic, and policy aspects of informatics, including telecommunications, the internet and related computing and media industries. He is currently a visiting scholar at Columbia University, Graduate School of Business where he is working on concepts of information infrastructure from the corporate to the international levels. He has written or edited nine scholarly books and numerous articles in studies of high technology industries, technology policy, and information systems (see http://is.lse.ac.uk/staff/liebenau).

G. Harindranath is a Senior Lecturer in Management Information Systems at Royal Holloway, University of London. He holds a doctorate in information systems from the London School of Economics. Hari's research interests include IS management, global IS issues, including information infrastructure policy, and IT and economic development. He is an associate editor for the Journal of Global Information Technology Management, and serves on the editorial board for the Journal of Global Information Management, and Transactions in International Information Systems. He is the current Vice President of International Relations for the Information Resources Management Association (IRMA) (see http://www. ms.rhul.ac.uk/hari). 